\title{
VIII.
}

\section{Ueber den Blutzucker.}

\author{
Von
}

Dr. E. Kälz,

Privatdocent in Marburg.

Bei meinen Studien über Diabetes habe ich, wo sich nur irgend dazu Gelegenheit bot, nicht verabsäumt, Blutuntersuchungen anzustellen. In Anbetracht der grossen Schwierigkeit, die die Beschaffung geeigneten Materials bietet, kann eine solche Untersuchung nur sehr langsam fortschreiten. Die bisher gewonnenen, zum Theil nicht unwichtigen Resultate halte ich für die Publication noch nicht reif genug. Eine vorläufige Mittheilnng ${ }^{1}$ ) von A. Cantani, Professor in Neapel, veranlasst mich jedoch, meine Erfahrungen über einen Punkt wenigstens zu veröffentlichen.

Schon früher (1865) hatte sich Cantani für die Existenz eines eigenthümlichen diabetischen Blutzuckers aus theoretischen Grïnden ausgesprochen. Den experimentellen Beweis für die Richtigkeit seiner Ansicht lieferte er erst später, unterstïtzt von Prof. Paladino. Das Verfahren, den Unterschied zwischen diabetischem Blutzucker und Traubenzucker festzustellen, war folgendes ${ }^{2}$ ):

Die betreffenden Diabeteskranken wurden mehrere Tage mit reichlicher Mehl- und Zuckerkost vorbereitet. Zu einer Zeit, wo vorhergegangene quantitative Analysen der einzelnen Harnentleerungen den grössten Zuckergehalt im Harn nachgewiesen hatten, wurde ein ergiebiger Aderlass gemacht.

Im Jahre 1872 wurde das Blut von 4 verschiedenen Kranken einzeln untersucht. Im Jahre 1873 wurde das Blut von 4 anderen

1) Ueber den diabetischen Blutzucker. Untersuchungen zur Naturlehre. Herausgegeben von Moleschott. 1875. XI. S. 443. Original.

2) Hinsichtlich einiger Details verweise ich auf das leicht zu beschaffende 
in demselben Augenblicke zur Ader gelassenen Kranken vereinigt der Prüfung unterworfen. Bei 2 Kranken wurde unter, bei 2 iiber $1 / 2$ pCt. Zucker durch Titriren mit Fehling'scher Lösung nachgewiesen. Die grösste Menge fand sich in dem vereinigten Blutserum der 4 Kranken vom Jahre 1873 vor, in welchem sich dieselbe auf 8 per Mille belief.

Die Untersuchung der optischen Eigenschaften des Blutzuckers, welche mittelst eines guten Soleil-Ventzke'schen Apparates ausgeführt wurde, führte $\mathrm{zu}$ dem interessanten Resultate, dass der diabetische Blutzucker nicht polarisirt. Sonst gleicht der diabetische Blutzucker dem des Harns in allen chemischen Reactionen vollkommen, wie er auch gleicherweise bei Hefezusatz Alkohol und Kohlensäure liefert. Der einzige wesentliche Unterschied besteht darin, dass der erstere optisch unwirksam ist.

Hinsichtlich seiner Ansicht über die Bedeutung dieser Thatsache für den diabetischen Krankheitsprocess und für das Wesen der Zuckerharnruhr selbst verweist Cantani auf sein im Erscheinen begriffenes Buch: Ueber Stoffwechsel-Pathologie und Therapie.

Angenommen, die Beobachtung wäre nicht nur für diese, sondern für alle Fälle von Diabetes unzweifelhaft richtig, kurz die Thatsache, der diabetische Blutzucker polarisire nicht, sei ganz sicher gestellt, so wäre es doch nach meiner Meinung wünschenswerth, ehe man diese Thatsache für den diabetischen Krankheitsprocess und für das Wesen der Zuckerharnruhr verwerthet, zuvor das optische Verhalten des normalen Blutzuckers zu prüfen. Er könnte sich ja vielleicht ebenfalls optisch inactiv erweisen und dann würden die auf jener Differenz beruhenden Speculationen doch wohl als hinfällig bezeichnet werden missen.

Bisher nahm man stillschweigend an, dass die im Menschenund Thierblut nachweisbare reducirend wirkende Substanz Traubenzucker sei. Ewald 1) stellte zuerst fest, dass die reducirend wirkende Substanz des normalen menschlichen Blutes das polarisirte Licht rechts dreht. Die gewiss dankenswerthe Beobachtung, deren Richtigkeit ich keineswegs bezweifle, verdient jedoch wiederholt zu werden, einmal, weil sie die erste und für das menschliche Blut einzige ist, dann aber auch, weil der Verdacht nicht ausgeschlossen ist, dass dem Blute, welches Ewald zu dieser Untersuchung verwandte, zum Theil der Inhalt des Ductus thoracicus beigemischt war.

1) Berl. klin. Wochenschr. 1875. No. 51 u. 52. 
M. Abeles ${ }^{1}$ ) wies nach, dass die aus 2 Liter Hundeblut gewonnene, reducirend wirkende Substanz wenn auch nur schwach, so doch deutlich rechts drehte.

Ich kann für Kalbsblut die Beobachtung ganz sicher bestätigen. Aus 3 Liter Kalbsblut konnte ich eine Flissigkeit isoliren, die stark reducirte und auf 25 C.-Ctm. eingeengt, eine Drehkraft besass, die $0,8 \mathrm{pCt}$. Traubenztcker entsprach.

Mit einer Ausnahme (Pat. Schlick) habe ich die Blutentziehungen bei einer Diät vorgenommen, in der Amylaceen reichlich vertreten waren.

Das Verfahren, welches ich bei der Untersuchung des Blutes auf Zucker beobachtete, war im Allgemeinen folgendes:

Das Blut wurde den diabetischen Patienten niemals durch den Aderlass, sondern stets rascher oder langsamer je nach dem Kräftezustand der Patienten durch den Schröpfkopf entzogen. Da die Messung doch keine genaue sein konnte, so sah ich davon ab, die Menge des in einer Sitzung entzogenen Blutes zu bestimmen; sie schwankte zwischen 50 und 120 C.-Ctm. Auf jeden Fall betrug die Gesammtmenge des von einem Patienten schliesslich gewonnenen Blutes nicht unter $300 \mathrm{C}$.-Ctm., in einem Falle mehr als das Doppelte (Pat. Schlick). Der Inhalt des Schröpfkopfes wurde rasch in eine Schale entleert, die etwa 1/2 Liter siedendes, mit einigen Tropfen Essigsäure angesäuertes Wasser enthielt, und das Ganze so lange gekocht, bis das Filtrat klar und leicht gelblich gefärbt abfloss. Der Niederschlag wurde noch mehrmals mit Wasser ausgekocht und schliesslich sorgf ältig ansgewaschen. Die vereinigten Filtrate wurden auf ein kleines Volumen (ca. 30 C.Ctm.) eingeengt. Da sich wiederum Coagula ausgeschieden hatten, so wurde nochmals filtrirt, der Niederschlag gut ausgewaschen und das Filtrat bis auf etwa 30 C.-Ctm. eingedampft. In der Flüssigkeit liess sich weder dureh Essigsäure noch dureh Salpetersäure Eiweiss nachweisen, wohl aber erhielt ich mit Salzsäure und Jodkaliumquecksilber noch einen nicht unbeträchtlichen Niedersehlag. Ich habe mich einige Male dieser von Brücke eingefübrten Methode mit gutem Erfolg bedient. Zur Entfärbung habe ich nie Thierkohle, sondern eine Lösung von Bleizucker benutzt. Die schliesslich resultirende Fliissigkeit wurde sowohl mit dem Polarisationsapparat von Soleil-Duboseq, als anch mit dem Polaristrobometer von Wild untersucht.

Die zur Untersuchung benutzten Diabeteskranken waren folgende:

1) Med. Jahrbücher. III. Heft. 1875.

Archiv fir experiment. Pathologie u. Pharmakologie. VI. Bd. 
1. Anna Elisabeth Schlick, 33 Jahr alt, Dienstmagd, schwere Form.

2. Georg Ast, 37 Jahr alt, Müller, schwere Form.

3. Julius Schmidt, 27 Jahr alt, Arbeiter, schwere Form.

4. Friedrich Happel, 60 Jahr alt, Fuhrmann, schwere Form.

5. Joh. Schneider, $38 \mathrm{Jahr}$ alt, Schuhmacher, leichte Form.

6. Heimrich Jahn, 24 Jahr alt, Oeconom, leichte Form.

Es gehörten somit 4 Fälle der schweren, 2 Fälle der leichten Form des Diabetes an.

Die erste Untersuchung wurde an der Pat. Schlick im Jahre 1873 ausgefiibrt. Bei strengem Ausschluss der Kohlenhydrate schied sie täglich im Durchschnitt 30 Grm. Zucker aus. Es kam mir damals darauf an, zu constatiren, ob der diabetische Blutzucker bei reiner Fleischdiät die Haupteigenschaften des Traubenzuckers zeige. Sowohl vorher als auch im Verlauf der Zeit, wo die Blutentziehungen gemacht wurden, hielt Pat. die strengste Fleischdiät inne. Innerhalb 3 Wochen wurden der Pat. sicher über 600 C.Ctm. Blut entzogen. Die hieraus in oben angegebener Weise erhaltene Fluissigkeit gab die gewöhnlichen Zuckerreactionen, drehte nach der Concentration sehr deutlich rechts und entwickelte, mit Hefe versetzt, Kohlensäure.

An Joh. Schneider gelang es mir sodann, auch für die leichte Form (bei gemischter Diät) dieselben Eigenschaften des Blutzuckers zu constatiren. Hiermit war meine Nengierde hinsichtich der Eigenschaften des diabetischen Blutzuckers vorläufig befriedigt. Die vorläufige Mittheilung: Cantani's veranlasste mich, die Beobachtung noch auf 4 weitere Fälle auszudehnen. In allen Fällen war das Endresultat: der diabetische Blutzucker polarisirt rechts. Bei den Patienten Ast, Schmidt und Happel wurde die Beobachtung sogar doppelt gemacht.

Ein Fall (Schmidt) kam zur Section. Wie ich an diesem Pat. zweimal zu verschiedenen Zeiten constatiren konnte, dass der Blutzucker rechts dreht, so gelang es mir auch posi mortem dasselbe Verhalten des Blutzuckers nachzuweisen. Der aus der Leber erhaltene Zucker drehte ebenfalls rechts. Ich stellte ferner aus einem Theil der Leber 0,45 Grm. reines Glykogen (pulverförmig, schneeweiss, frei von Stickstoff und Asche) dar. Mit reinem menschlichem Parotidenspeichel versetzt gab das übrigens rechts drehende Glykogen reducirenden Zucker, der ebenfalls rechts drehte. ${ }^{1}$ )

1) Im vergangenen Jahre hatte ich Gelegenheit, das Blut eines an Diabetes verstorbenen Mädchens (15 Jahre alt) zu untersuchen. Die Form des Diabetes kann ich nicht angeben, da ich den Fall intra vitam nicht beobachtete. Der Blutzucker erwies sich auch hier als optisch wirksam. 
Wenn ich allen Beobachtungen Rechnung trage, so musste ich z. B. an dem Apparat von Soleil-Duboseq den Zeiger um 3--6 Theilstriche uiber den Nullpunkt hinausschieben. Ein Irrthum ist hier also nicht wohl möglich. $O b$ die Drehkraft des diabetischen Blutzuckers geringer ist, als die des diabetischen Harnzuckers, kann ich aus meinen Beobachtungen nicht folgern. Ich habe das bis jetzt gewonnene Material aufbewahrt und hoffe durch weitere Beobachtungen den diabetischen Blutzucker in Substanz darstellen und das optische Verhalten desselben mit dem des Traubenzuckers vergleichen zu können.

Der Gïte des Herrn Dr. Salomon verdanke ich ein reines Präparat von Glykogen, das aus der Leber eines Kindes, an dem die Perforation gemacht war, dargestellt wurde. $\left.{ }^{1}\right)$ Der durch Fermentirung hieraus hervorgegangene Zucker drehte ebenfalls rechts.

Wenn man Kaninchen, die volle 6 Tage gehungert haben, mit Traubenzucker, Inulin, Fruchtzucker, Rohrzucker, Milchzucker, Glycerin fiuttert, so findet man nach Einfuhr jeder einzelnen dieser Substanzen ein Glykogen vor, das, mit Parotidenspeichel saccharificirt, einen rechtsdrehenden Zucker liefert.

Nach diesen Erfahrungen kann es sich jetzt wohl nur noch darum handeln, den sicheren Nachweis zu liefern, dass der diabetische Blatzucker in gewissen Fällen inactiv ist. Gewagt dürfte es sein, die physikalische Differenz, wenn sie sich durch kritische Untersuchungen für gewisse Fälle als richtig erweisen sollte, schon jetzt für das Wesen der Zuckerruhr verwerthen za wollen.

1) CentralbI. f. d. med. Wissensch. Jahrg. XII. (1874) S. 738. 